\title{
Müdigkeit bleibt noch lange nach der Chemo
}

\begin{abstract}
Mehr als drei Jahre nach einer Chemotherapie gegen Brustkrebs halten Fatigue-Symptome an - deutlich länger als angenommen. Dies geht aus einer Studie niederländischer und US-amerikanischer Forscher hervor.
\end{abstract}

\begin{abstract}
S ie hatten drei Gruppen von Patien$\int$ tinnen mit Mammakarzinomen der Stadien 0-II untersucht. 103 Patientinnen, die eine Chemotherapie mit oder ohne Strahlentherapie erhalten hatten (Gruppe 1), 102, die nur bestrahlt worden waren
\end{abstract}

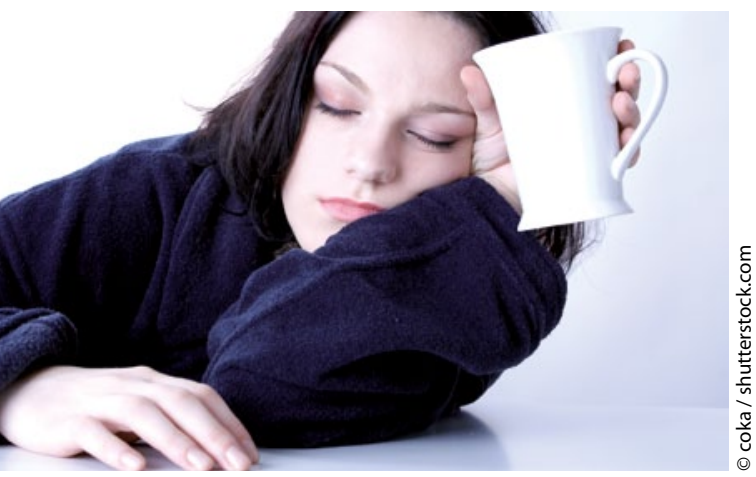

Chronisch stark erschöpft: Das Problem haben Frauen nach MammakarzinomBehandlung länger als gedacht.
(Gruppe 2) und 193 Kontrollpatientinnen, die nicht an Krebs erkrankt waren (Gruppe 3).

Sechs und 42 Monate nach Ende der Behandlung wandten die Wissenschaftler das „Fatigue Symptom Inventory“ und die „Profile of Mood States Fatigue Scale“ an. Erwartet wurde, dass die chemotherapierten Patientinnen nach der Behandlung stärker unter Fatigue leiden, die Beschwerden sich aber mit der Zeit dem Niveau in den anderen beiden Gruppen angleichen würden. Doch weit gefehlt: Nicht nur gingen die Symptome nicht zurück, es gab sogar Anzeichen dafür, dass sie sich während der drei Jahre zwischen erster und zweiter Untersuchung verschlechtert hatten. So nahm beispielsweise die Schwere der Fatigue in der Chemotherapie-Gruppe signifikant $\mathrm{zu}(\mathrm{p}=0,007)$, nicht aber in den beiden anderen Gruppen. Etwa jede dritte Frau, die eine Chemothe- rapie durchgemacht hatte, befand sich im pathologischen Bereich, in den Gruppen 2 und 3 nur rund jede fünfte. Sämtliche untersuchten Fatigue-Parameter fielen nach einer Chemotherapie zu beiden Testzeitpunkten schlechter aus.

Fazit: „Diese Ergebnisse haben wichtige klinische Konsequenzen“, schrieben die Autoren. Brustkrebs-Patientinnen müssten nach einer Chemotherapie einem kontinuierlichen posttherapeutischen Monitoring auf Fatigue unterzogen werden. Auch sollte man ihnen nicht sagen, ihre Müdigkeit werde sich im Lauf der Zeit bessern, sondern ihnen reinen Wein einschenken: Es könne sogar sein, dass statt einer Verbesserung eine Verschlechterung eintrete. „Zugleich sollten sie über Maßnahmen gegen die Fatigue unterrichtet werden", so die Forscher, zum Beispiel kognitive Verhaltenstherapie oder körperliches Training.

Robert Bublak

Goedendorp MM et al. Prolonged impact of chemotherapy on fatigue in breast cancer survivors. Cancer. 2011 Nov 15. doi: 10.1002/ cncr.26226. [Epub ahead of print].

\section{Betroffene sehen brusterhaltende Operation kritischer als Ärzte}

Kognitive Einbußen, gestörte Sexualität, Hautveränderungen und Schmerzen sind einige der Beschwerden, über die Patientinnen nach brusterhaltender Therapie eines Mammakarzinoms in einer anonymen Befragung klagen. Auch die kosmetischen Ergebnisse werden erstaunlich kritisch beurteilt.

\footnotetext{
- ür die Studie hatte ein amerikanisches

- Team 354 Brustkrebs-Patientinnen anonym befragt, die eine brusterhaltende Therapie - Lumpektomie plus adjuvante Radiotherapie, meist plus Chemotherapie - überstanden hatten. Sie waren bei Diagnose median 48, zum Zeitpunkt der Umfrage 52 Jahre alt gewesen. Als Instrument diente ein Onlinewerkzeug zur Entwicklung von Nachsorgeplänen im Internetangebot von OncoLink (www.livestrongcareplan.org), einer Website des Abramson Cancer Center der University of Pennsylvania, wo auch nach unerwünschten Effekten der Therapie gefragt wird.

$62 \%$ der Patientinnen gaben kognitive Einbußen durch die Therapie an, über
}

eine veränderte Sexualität klagten $52 \%$, über Hautveränderungen im Bestrahlungsgebiet $48 \%$. Weitere Beschwerden waren chronische Schmerzen, Taubheit und Kribbeln im ipsilateralen Arm (35\%), Osteopenie/Osteoporose (35\%), kardiopulmonale Störungen (12\%) und Lymphödeme (19\%).

Unerwartet schlecht beurteilten die Frauen das kosmetische Ergebnis der brusterhaltenden Behandlung. Als exzellent oder gut stuften nur rund $70 \%$ den Zustand der operierten Brust ein, die übrigen bezeichneten ihn als allenfalls ausreichend $(24 \%)$ oder schlecht $(5 \%)$. In anderen Studien, in denen Ärzte das Ergebnis nach brusterhaltender Therapie beurteilt hatten, lag der Anteil von Frauen mit exzellentem oder gutem Resultat hingegen bei bis zu $95 \%$.

Fazit: „Soweit wir wissen, ist dies die erste Studie, die Auskunft darüber gibt, wie die Betroffenen selbst die Ergebnisse brusterhaltender Krebstherapie in einer anonymen Erhebung einschätzen", schrieben die Wissenschaftler um C. E. Hill-Kayser. Zwar neigten unzufriedene Patientinnen vielleicht eher dazu, sich an solchen Umfragen zu beteiligen. Doch dürfe man den Wert der Resultate für die Beratung vor einer Brustkrebstherapie und für eine umfassende Nachsorge nicht übersehen: „Speziell bei Brustkrebs-Patientinnen beeinflusst die eigene Einschätzung der Therapieresultate nachweislich das Gesamtüberleben."

Robert Bublak

Hill-Kayser CE et al. Cosmetic outcomes and complications reported by patients having undergone breast-conserving treatment. Int J Radiat Oncol Biol Phys. 2011 Dec 2. [Epub ahead of print]. 